\title{
Mefloquina no tratamento da leishmaniose cutânea em uma área endêmica de Leishmania (Viannia) braziliensis
}

\author{
Efficacy of mefloquine in the treatment of skin leishmaniasis in an \\ endemic area of Leishmania (Viannia) braziliensis
}

\author{
Victor Alberto Laguna-Torres, Carlos A.C. Silva, Dalmo Correia, Edgard M. Carvalho, \\ Albino V. Magalhães e Vanize de Oliveira Macêdo
}

\begin{abstract}
Resumo O objetivo deste trabalho foi avaliar a eficácia da mefloquina numa região endêmica de leishmaniose cutânea por Leishmania (Viannia) braziliensis, considerando que esta droga de administração oral, eficaz no tratamento da malária, com meia vida prolongada e efeitos colaterais pouco freqüentes poderia ser menos tóxica e de mais fácil administração, quando comparada com os antimoniais pentavalentes. Em Corte de Pedra, no litoral sul do Estado da Bahia, foram tratados, aleatoriamente, dez pacientes portadores de lesões leishmanióticas, subdivididos em dois grupos. O primeiro grupo recebeu mefloquina pela via oral, dose de $250 \mathrm{mg} / \mathrm{dia}$, durante seis dias, repetindo-se o mesmo esquema após intervalo de três semanas. O segundo grupo recebeu antimoniato de meglumina (Glucantime ${ }^{\circledR}$ ) diariamente, pela via endovenosa, na dose de $20 \mathrm{mg} / \mathrm{kg}$ por 20 dias. Do grupo da mefloquina só um paciente apresentou cicatrização depois do segundo ciclo. Um desses, com quatro lesões apresentou nova lesão durante o primeiro ciclo de tratamento. A evolucão dos outros três foi lenta sendo que em nove semanas nenhum deles tinha cicatrizado as úlceras que permaneciam com grande infiltração e sinais evidentes de atividade. O grupo tratado com Glucantime $\AA$ apresentou evidente melhora.
\end{abstract}

Palavras-chaves: Mefloquina. Leishmaniose tegumentar. Glucantime.

\begin{abstract}
The aim of this study was to evaluate the efficacy of mefloquine in the treatment of skin leishmaniasis in patients infected with Leishmania (Viannia) braziliensis at an endemic region. Mefloquine is an oral drug effective against malaria with a prolonged half-life, less toxicity and easier administration than pentavalent antimonials. At Corte de Pedra in the Southern litoral of Bahia State, two randomized groups of ten patients with leishmaniasis were treated. The first group was treated with oral mefloquine, $250 \mathrm{mg}$ per day in a single dose for six days and repeated three weeks later. The second group received meglumine antimoniate (Glucantime $\mathbb{B}$ ), $20 \mathrm{mg} / \mathrm{kg}$ daily administered intravenously for 20 days. Only one patient in the group treated with mefloquine showed evidence of clinical success. During treatment, one patient with four lesions developed a new lesion. The other three patients with clinical leismaniasis did not show evidence of clinical success after nine weeks of treatment. The group treated with Glucantime ${ }^{B}$ showed evident clinical improvement of the skin lesions.
\end{abstract}

Key-words: Mefloquine. Tegumentary leishmaniasis. Glucantime.

\footnotetext{
Núcleo de Medicina Tropical e Nutrição da Universidade de Brasília, Brasília, DF, Faculdade de Medicina do Triângulo Mineiro, Uberaba MG e Universidade Federal da Bahia, Salvador, BA.

Endereço para correspondência: Dr. Victor Alberto Laguna-Torres. Los Amancaes 381, Urb I, Jardines Virú, Callao 2, Lima, Peru. Fax: $00511433-0081$.

E-mail: alaguna@oge.sld.pe

Recebido para publicação em 7/1/99.
} 
Os antimoniais pentavalentes são as drogas de escolha para o tratamento da leishmaniose, sendo eficazes em $80 \%$ dos pacientes. Apresentam problemas como toxicidade, administração parenteral e dificuldade para estabelecer quantidades variáveis de antimônio que se encontra na solução utilizada ${ }^{4}{ }^{9}$.

A mefloquina é uma droga de administração oral, com provada eficácia na malária, com meia vida prolongada e efeitos colaterais diversos, pouco freqüentes ${ }^{6}$. Gómez e cols ${ }^{5}$, em 1995, no Equador, trataram 16 pacientes com mefloquina mostrando $100 \%$ de cura antes de 6 semanas.

O objetivo deste trabalho foi avaliar a eficácia da mefloquina numa região endêmica de leishmaniose cutânea causada por Leishmania (Viannia) braziliensis ${ }^{11}$.

\section{PACIENTES E MÉTODOS}

O estudo foi desenvolvido no Centro de Saúde de Corte de Pedra (CSCP), Tancredo Neves, localizado no litoral sul do Estado da Bahia, entre janeiro e abril de 1996.

Foram incluídos, aleatoriamente, dez pacientes divididos em dois grupos. O primeiro recebeu mefloquina oral, um comprimido de $250 \mathrm{mg} /$ dia por seis dias em duas séries com intervalos de três semanas. O segundo grupo recebeu Glucantime ${ }^{\circledR} 20 \mathrm{mg} / \mathrm{kg} /$ dia EV por 20 dias.

Os critérios de inclusão foram idade entre 12 e 45 anos, moradia permanente na área endêmica, úlcera clinicamente compatível com leishmaniose, e pelo menos um dos seguintes exames diagnósticos positivos: intradermorreação de Montenegro (IDRM), sorologia IFI > 1:32, esfregaço, histopatologia ou cultura. $\mathrm{O}$ antígeno para a IDRM foi fornecido pelo laboratório de Imunologia do Hospital Prof. Edgar Santos da Universidade Federal da Bahia (UFBA). Definiuse cura clínica como a presença de reepitelização sem sinais inflamatórios como edema, descamação, ou eritema, na nona semana após o início do tratamento. Os pacientes em tratamento com Glucantime ${ }^{\circledR}$ receberam a primeira dose no CSCP, e depois domiciliarmente, quando comprovada a existência de um agente de saúde treinado na aplicação endovenosa do medicamento; quando necessário o medicamento foi aplicado no CSCP.

Os pacientes do grupo da mefloquina receberam a primeira e a quinta dose no CSCP. O acompanhamento realizou-se, semanalmente, por cinco semanas, considerando-se o tamanho e a infiltração da lesão, o aparecimento de novas lesões, e os efeitos colaterais de cada um dos grupos em tratamento.

$\mathrm{Na}$ quinta semana depois de iniciado o tratamento, os pacientes foram avaliados por um segundo observador da UFBA. Na nona semana foram reavaliados pela nossa equipe. Os pacientes do grupo de mefloquina não curados foram imediatamente tratados com Glucantime $\AA$, no esquema definido anteriormente.

\section{RESULTADOS}

Do grupo da mefloquina só um paciente apresentou cicatrização completa de lesão, depois do segundo ciclo de tratamento. Um dos doentes, com quatro lesões, apresentou nova lesão durante o primeiro ciclo de tratamento. A evolução dos outros três foi lenta, sendo que em nove semanas nenhum deles tinha cicatrizado as úlceras, que permaneciam com grande infiltração e sinais evidentes de atividade. $O$ grupo tratado com Glucantime ${ }^{\circledR}$ apresentou evidente melhora, incluindo uma paciente com seis lesões comprometendo as mamas. Um dos pacientes deste grupo só voltou na primeira semana e foi considerado como abandono. Outro não foi avaliado na última semana.

Dos efeitos colaterais apresentados, dois pacientes do grupo de mefloquina relataram tonturas no primeiro ciclo de tratamento.

$\mathrm{Na}$ Tabela 1, apresentamos algumas características clínicas e laboratoriais dos pacientes.

\section{DISCUSSÃO}

O desenvolvimento de drogas leishmanicidas de baixa toxicidade e de administração oral traria grandes vantagens sobre os antimoniais. A mefloquina cumpre com duas condições, administração por via oral e baixa toxicidade, mas não conseguimos repetir o sucesso relatado por Gómez e cols ${ }^{5}$, que obtiveram cura em $100 \%$ dos casos.
A mefloquina possui um tempo de meia vida de 21 dias e isto foi determinante para o seguimento de nossos pacientes, uma vez que depois de três semanas de administrado o tratamento, os seus efeitos ainda teriam de ser avaliados. Por isso, acompanhamos os doentes por nove semanas; mesmo assim, obtivemos cura em apenas um, o que poderia ser interpretado 
Tabela 1 - Características clínico-laboratoriais dos pacientes tratados em Corte de Pedra, Bahia. 1996.

\begin{tabular}{|c|c|c|c|c|c|c|c|c|}
\hline \multirow[b]{2}{*}{ Caso } & \multirow[b]{2}{*}{ Idade } & \multicolumn{3}{|c|}{ Lesões } & \multirow[b]{2}{*}{ Sorologia } & \multicolumn{2}{|c|}{ Parasitas } & \multirow[b]{2}{*}{ Tratamento } \\
\hline & & $\mathrm{n}^{\circ}$ & localização & duracão (dias) & & histopatologia & cultura & \\
\hline 1 & 28 & 1 & $\mathrm{MI}$ & 90 & $1: 16$ & + & + & mefloquina \\
\hline 2 & 25 & 1 & MI & 30 & $1: 64$ & + & + & mefloquina \\
\hline 3 & 19 & 4 & $\mathrm{Ml}+$ tronco & 30 & $1: 64$ & - & & mefloquina \\
\hline 4 & 13 & 1 & MS & 15 & $1: 64$ & + & & mefloquina \\
\hline 5 & 17 & 1 & MI & 60 & $1: 64$ & + & & mefloquina \\
\hline 6 & 13 & 1 & MI & 45 & $(-)$ & - & + & glucantime \\
\hline 7 & 40 & 2 & MI & 18 & $1: 16$ & - & & glucantime \\
\hline 8 & 22 & 6 & $\mathrm{MS}+$ tronco & 60 & $1: 64$ & - & & glucantime \\
\hline 9 & 35 & 1 & MI & 30 & NR & - & + & glucantime \\
\hline 10 & 29 & 3 & $\mathrm{MI}$ & 15 & $1: 64$ & + & + & glucantime \\
\hline
\end{tabular}

como cura espontânea, já bem documentada na leishmaniose tegumentar americana ${ }^{13}$.

Vários estudos têm procurado encontrar alternativas terapêuticas na leishmaniose ${ }^{21012}$. O sucesso relatado por Gómez e cols ${ }^{3}$ poderia ser explicado por ter ele trabalhado com outra cepa diferente de Leishmania, a Leishmania (Viannia) panamensis, que sabidamente responde melhor ao tratamento.

Tratamentos inadequados condicionam maior risco de se desenvolver lesão mucosa ${ }^{8}$, o que poderia suceder com a mefloquina. Os antimoniais, de reconhecida eficácia ${ }^{7}$, só poderiam ser substituídos por uma droga que apresentasse uma percentagem de cura superior ou, pelo menos, igual à deles.

\section{AGRADECIMENTOS}

O autor agradece ao Núcleo de Medicina Tropical e Nutrição de Brasília e à CAPES pelo apoio durante a realização deste trabalho.

\section{REFERÊNCIAS BIBLIOGRÁFICAS}

1. Carvalho EM, Correia Filho D, Bacellar O, Almeida PR, Lessa $\mathrm{H}$, Rocha $\mathrm{H}$. Characterization of the immune responses in subjects with self-healing cutaneous leishmaniasis. American Journal of Tropical Medicine and Hygiene 53:273-277, 1995.

2. Correia D, Macêdo VO, Carvalho EM, Barral A, Magalhães AV, Abreu MVA, Orge MGO, Marsden P. Estudo comparativo entre antimoniato de meglumina, isotianato de pentamidina e sulfato de aminosidine, no tratamento de lesões cutâneas primárias causadas por Leishmania (Viannia) braziliensis. Revista da Sociedade Brasileira de Medicina Tropical 29:447-453, 1996.

3. Costa JML, Vale K, França F, Saldanha C, Silva J, Lago E, Marsden PD, Magalhães A, Conceição ES, Serra Neto A, Galvão C. Cura espontânea da leishmaniose causada por Leishmania Viannia brasileinsis em lesões cutâneas. Revista da Sociedade Brasileira de Medicina Tropical 23:205-208, 1990.

4. Franco MA. Determinação de antimoniais (SbIll e Sb V) em fármacos. Tese de Mestrado, Universidade de Brasília, Brasília, DF, 1992.

5. Gómez LE, Andrial M, Hosokawa A, Nonade S, Hashiguchi $Y$. Oral treatment of new world cutaneous leishmaniasis with mefloquine and artesunate in Ecuador: a preliminary clinical trial. Japanese Journal of Tropical Medicine and Hygiene 23:151-157,1995.

6. Kaltrarine P, Stephen H, Rex B. Mefloquine. A review of its antimalarial activity, pharmacocinetics properties and therapeutic efficacy. Drugs 45: 430-475, 1993.

7. Llanos-Cuentas E. Estudo clínico evolutivo da leishmaniose em área endêmica de Leishmania braziliensis braziliensis. Três Braços - Bahia. Tese de Mestrado, Universidade de Brasília, Brasília, DF, 1992.

8. Llanos-Cuentas E, Marsden PD, Cuba C, Barreto A, Campos M. Possible risk factors in development of mucosal lesion in leishmaniasis. The Lancet II: 295,1984.

9 Marsden PD. Pentavalent antimonials: old drug for new diseases. Revista da Sociedade Brasileira de Medicina Tropical 18: 187-198, 1985.

10. Pradinaud R, Servans G, Sainte-Marie D, Girardeau I, Gotz W, Will F. Bilan de 10 années de traitement de la leishmaniose tégumentaire par la pentamidine en Guyane Française. Nouvelles Dermatologie 10:456-611, 1991.

11. Rosa AC, Cuba CC, Vexenat JA, Barreto AC. Predominance of Leishmania braziliensis in the regions of Três Braços and Corte de Pedra, Bahia, Brazil. Trasactions of the Royal Society of Tropical Medicine and Hygiene 82: 409-410,1998. 
12. Talhari S, Sardinha JCG, Schettini APM, Aria J, Naiff RD. Tratamento da leishmaniose tegumentar americana: resultados preliminares com a pentamidina. Anais Brasileiros de Dermatologia 60:361-364, 1985. 